\title{
Radial distribution of the diffuse gamma-ray emissivity in the galactic disk
}

\author{
Ruizhi Yang* \\ Max-Planck-Institut für Kernphysik, \\ P.O. Box 103980, 69029 Heidelberg, Germany
}

Felix Aharonian

Max-Planck-Institut für Kernphysik, P.O. Box 103980, 69029 Heidelberg, Germany Dublin Institute for Advanced Studies, 31 Fitzwilliam Place, Dublin 2, Ireland.

Carmelo Evoli

Gran Sasso Science Institute, 7 viale Francesco Crispi, 67100 LAquila (AQ), Italy

(Dated: Received: / Accepted:)

\begin{abstract}
The Fermi-LAT data accumulated over 7 years of $\gamma$-ray observations, together with the high resolution gas ( $\mathrm{CO} \& \mathrm{HI}$ ) and the dust opacity maps, are used to study the emissivity of $\gamma$-rays induced by interactions of cosmic rays (CRs) with the interstellar medium. Based on the dust opacity templates, the $\gamma$-ray emissivity was measured for 36 segments of the Galactic plane. Furthermore, the $\gamma$-ray emissivity was evaluated in six Galactocentric rings. Both the absolute emissivity and the energy spectra of $\gamma$-rays derived in the interval $0.2-100 \mathrm{GeV}$ show significant variations along the galactic plane. The density of CRs, derived under the assumption that $\gamma$-rays are predominately produced in CR interactions with the interstellar gas, is characterised by a strong radial dependence. In the inner Galaxy the CR density substantially exceeds the density in the outer parts of the Galaxy: by a factor of a few at $10 \mathrm{GeV}$, and by more than an order of magnitude at $1 \mathrm{TeV}$. Remarkably, the energy distribution of CRs appears to be substantially harder than the energy spectrum obtained from direct measurements of local CRs. At the same time, the flux and the energy spectrum of multi-GeV protons derived from $\gamma$-ray data in the outskirts of the Galaxy is quite close to the measurements of local CRs.
\end{abstract}

PACS numbers: 95.85.Ry; 98.70.Sa

\section{INTRODUCTION}

There is a general consensus within the cosmic ray (CR) physics community that up to the so-called knee, a distinct spectral feature around $10^{15} \mathrm{eV}$, CRs are produced in galactic sources, presumably in supernova remnants [for a review, see e.g. 1-3] The direct studies of CRs, which include measurements of the energy spectrum, the mass composition and arrival directions of particles, provide an important but yet not decisive information about the production sites of CRs and their propagation in the Galaxy. The diffusion of CRs in turbulent magnetic fields erases, to a large extent, the information on the distribution of $\mathrm{CR}$ accelerators. The energy dependent-diffusion of CRs also significantly modifies the initial (acceleration) spectra of CRs. In this regard, $\gamma$ rays, the secondary products of interactions of CRs, tell us more information about the origin of CRs. Namely, while the discrete $\gamma$-ray sources elucidate the locations of individual CR accelerators, the diffuse $\gamma$-ray component contains an information about the global distribution of CR factories, as well as about the CR propagation throughout the Galaxy.

\footnotetext{
* ryang@mpi-hd.mpg.de

† felix.aharonian@mpi-hd.mpg.de

¥ carmelo.evoli@gssi.infn.it
}

Historically, since the first $\gamma$-ray measurements, the study of the diffuse gamma-radiation of the Galactic Disk has been among the major objectives of gamma-ray astronomy. Unfortunately, this component of radiation is contaminated by contributions of weak but numerous unresolved $\gamma$-ray sources. Therefore its extraction is a quite difficult task. This can explain the relatively slow progress in this area. The improvement of the flux sensitivity by Fermi-LAT, compared to the previous gammaray missions, by an order of magnitude, and, perhaps more importantly, the extension of the dynamical range of measurements over more than three decades, from 100 $\mathrm{MeV}$ to $\geq 100 \mathrm{GeV}$, promise a breakthrough regarding both the removal of the background caused by individual sources and separation of the leptonic (bremsstrahlung and inverse Compton) and hadronic ( $p p$ and $p A$ ) radiation components.

Over the last several years, a significant progress has been achieved also towards the knowledge of the gas distribution in our Galaxy. This is a key issue for extraction of the diffuse galactic $\gamma$-ray emission and for derivation of the spectral and spatial distributions of parent CR particles. In this regard, one should mention the recent reports of the Planck Collaboration on the dust opacity maps which are complementary to the $\mathrm{CO}$ and 21 $\mathrm{cm}$ measurements of the molecular and atomic hydrogen distributions, respectively.

In this paper, we report the results of our study of the 
diffuse $\gamma$-ray emission of the Galactic Disk based on the Fermi-LAT observations, and briefly discuss the implications of these results, in particular in the context of spatial and energy distributions of CRs in the Galactic Disk.

\section{THE GAS TRACERS}

The traditional tracers of the hydrogen in the atomic and molecular forms are the $21 \mathrm{~cm} \mathrm{HI}$ and $2.6 \mathrm{~mm}$ CO lines, respectively. In this paper we use the data from CO galactic survey of Dame et al. [4] with the CfA $1.2 \mathrm{~m}$ millimetre-wave Telescope, and the Leiden/Argentine/Bonn (LAB) Survey on HI gas. For the $\mathrm{CO}$ data, we use the standard assumption of a linear relationship between the velocity-integrated CO intensity, $W_{\mathrm{CO}}$, and the column density of molecular hydrogen, $\mathrm{N}\left(\mathrm{H}_{2}\right)$. The conversion factor $X_{\mathrm{CO}}$ may be different in different parts of the galaxy, therefore we leave this quantity as a free parameter in the likelihood fitting below.

For the HI data we use the equation

$$
N_{H I}\left(v, T_{s}\right)=-\log \left(1-\frac{T_{B}}{T_{s}-T_{b g}}\right) T_{s} C_{i} \Delta v,
$$

where $T_{b g} \approx 2.66 \mathrm{~K}$ is the brightness temperature of the cosmic microwave background radiation at $21 \mathrm{~cm}$, and $C_{i}=1.83 \times 10^{18} \mathrm{~cm}^{2}$. In the case when $T_{B}>T_{s}-5 \mathrm{~K}$, we truncate $T_{B}$ to $T_{s}-5 \mathrm{~K} ; T_{s}$ is chosen to be $150 \mathrm{~K}$. The systematic uncertainties due to the different spin temperatures are discussed in ref.Casandjian [5]. The effect, however, is quite small in most regions of the sky.

For different reasons, the neural gas cannot be always traced by $\mathrm{CO}$ and $\mathrm{HI}$ observations. In such cases (e.g. in optically thick clouds), the infrared emission from cold interstellar dust provides an alternative and independent measurements of the gas column density. To find it, we need a relation between the dust opacity and the column density. According to Equation (4) of ref.Planck Collaboration et al. [6],

$$
\tau_{M}(\lambda)=\left(\frac{\tau_{D}(\lambda)}{N_{H}}\right)^{d u s t}\left[N_{H \mathrm{I}}+2 X_{C O} W_{C O}\right],
$$

where $\tau_{M}$ is the dust opacity as a function of the wavelength $\lambda,\left(\tau_{D} / N_{H}\right)^{d u s t}$ is the reference dust emissivity measured in low $-N_{H}$ regions, $W_{C O}$ is the integrated brightness temperature of the $\mathrm{CO}$ emission, and $X_{C O}=$ $\mathrm{N}_{\mathrm{H}_{2}} / W_{\mathrm{CO}}$ is the $\mathrm{H}_{2} / \mathrm{CO}$ conversion factor. The substitution of the latter into Equation (2) gives

$$
N_{H}=N_{H \mathrm{I}}+2 N_{H_{2}}=\tau_{m}(\lambda)\left[\left(\frac{\tau_{D}(\lambda)}{N_{H}}\right)^{d u s t}\right]^{-1} .
$$

Here for the dust emissivity at $353 \mathrm{GHz}$, we use $\left(\tau_{D} / N_{H}\right)_{353 \mathrm{GHz}}^{\text {dust }}=1.18 \pm 0.17 \times 10^{-26} \mathrm{~cm}^{2}$ taken from Table 3 of ref.Planck Collaboration et al. [6] .
General, the dust opacity is considered as a robust and reliable estimate of the gas column density. On the other hand, the dust opacity maps do not contain an information on the distance. In order to investigate the Galactocentric radial distribution of CRs we need to divide the gas distribution into different rings around the GC. For this purpose we use the following relation

$$
V_{L S R}=R_{\odot}\left(\frac{V(R)}{R}-\frac{V_{\odot}}{R_{\odot}}\right) \sin (l) \cos (b),
$$

where $R$ is the galactocentric distance, $V(R)$ is the Galactic rotational curve, and $l$ and $b$ are the galactic coordinates in the line of sight (LOS). We adopt the rotational curve parametrised in ref.Clemens [7]; $V_{\odot}$ and $R_{\odot}$ are fixed to $220 \mathrm{~km} / \mathrm{s}$ and $8.5 \mathrm{kpc}$, respectively. By applying this to both $\mathrm{CO}$ and $\mathrm{HI}$ data, we can transform the velocity information into galactocentric distance of the gas. Below the $\mathrm{CO}$ and $\mathrm{HI}$ data are binned into six distance intervals: $[1,2],[2,4],[4,6],[6,8],[8,12]$ and $[12,25]$ kpc. Due to the limited sky coverage by the CO surveys, we limit our study by the regions with $|b|<5^{\circ}$. It should be noted that Eq, 4 allows emission from the forbidden velocity zones in the $\mathrm{CO}$ and $\mathrm{HI}$ data. The reason could be the non-circular motion of gas. This component contains only small fraction of total gas, therefore for simplicity we assigned it into the local rings. In the GC and anticenter directions, the velocity resolution does not allow us to determine the distance. Therefore in the gamma ray likelihood fittings we mask out the 10 degree regions around the GC and anti-center.

As mentioned above, in some cases the HI and CO measurements cannot trace the neutral gas. For the missing "dark" gas, we use the dust data to derive the residual templates. We fit the dust opacity maps as a linear combination of $\mathrm{HI}$ and $\mathrm{CO}$ maps, and, in this way, find the residual map as the "dark" gas template. Then we iterate this fit by including the "dark" gas template until a convergence is achieved. To account for the $X_{C O}$ variation in different regions of the Galaxy, we perform the fitting in different segments of the galactic plane, each of $10^{\circ} \times 10^{\circ}$ angular size. This method is similar to the derivation of the $E(B-V)_{\text {res }}$ templates used by the Fermi LAT collaboration Casandjian [5], but instead of the extinction maps we use the dust opacity maps. The detailed study of the "dark" gas distribution is beyond the scope of this paper. But, in any case, the gamma-ray fitting below shows that in our ROI the "dark" gas has only minor impact on the final results.

\section{GAMMA RAY DATA}

In this study we included the observations of FermiLAT accumulated over a period of approximately 7 years (MET 239557417 - MET 451533077), and selected all events with energies above $100 \mathrm{MeV}$. For the data reduction, we use the standard LAT analysis software pack- 
age $v 10 r 0 p 51$. In order to reduce the effect of the background caused by the Earth albedo, we excluded from the analysis the time intervals when the Earth was in the field-of-view (more specifically, when the centre of the field-of-view was more than $52^{\circ}$ above the zenith), as well as the time intervals when the parts of the ROI were observed at zenith angles $>90^{\circ}$. The spectral analysis was performed based on the P8R2_v6 version of the post-launch instrument response functions (IRFs). Both the front and back converted photons have been selected. Since the galactic diffuse model provided by the Fermi collaboration 2 already contains the emission component from the CR-gas interactions, we do not include it in the analysis. In the Galactic plane, in the energy interval of interest between $100 \mathrm{MeV}$ and $100 \mathrm{GeV}$, the diffuse $\gamma$-ray emission is dominated by the channel of decay of neutral pions produced at interactions of CR protons and nuclei with the interstellar gas, with a significantly smaller contribution from the Inverse Compton (IC) scattering of relativistic electrons [see e.g. 8]. The emissivity of the pion decay gamma rays is proportional to both the CR and gas densities. Thus we use the dust opacity map and gas maps based on the $\mathrm{CO}$ and $\mathrm{HI}$ observations as the "pion-decay template". The IC templates has been calculated by using the Galprop code 3 [9], based on the information regarding the CR electrons and the interstellar radiation field (ISRF). Furthermore, we included an isotropic template to take into account the foregrounds like the contamination caused directly by CRs, and by the isotropic extragalactic $\gamma$-ray background. Finally, we used the 3FGL catalogue [10] of the Fermi-LAT collaboration, to exclude contributions from the the resolved point-like $\gamma$-ray sources.

For evaluation of CR density in different regions of the Galactic Disk, the standard Fermi-LAT tools gtlike are not suitable for this task. To perform the analysis, we first divide the entire $0.1-200 \mathrm{GeV}$ region into 10 equal in logarithmic scale energy bins, and generate the count maps and exposure maps in each energy bin by using the standard routine gtbin and gtexpcube2. Then we generate the source map by convolving the point sources and the diffuse emission templates with the point spread function (PSF) in each energy bin. At low energies, to account for the larger PSF, we produce the source map in a larger area compared with the ROI. For example, in the analysis below with dust templates, where each ROI is a $10^{\circ} \times 10^{\circ}$ square, we generate the source map for diffuse templates with a size of $30^{\circ} \times 30^{\circ}$. The likelihood function has the same form as the one used in gtlike, $\log (L)=\sum_{k_{i}} \log \left(\mu_{i}\right)-\mu_{i}-\log \left(k_{i} !\right)$, where $k$ is the number of photons in the $i$-th bin in the counts map, and $\mu_{i}$ is the predicted number of photons within a particular linear combination of the templates. We use iminuit 4 to minimise the $-\log (\mathrm{L})$ function and derive the best fit parameter and their error distributions.

\section{A. The analysis with dust templates}

We divided the plane into thirty six $10^{\circ} \times 10^{\circ} \mathrm{seg}-$ ment. In each patch, we fit the $\gamma$-ray emission using the catalog point sources, the dust opacity template, the IC template and the isotropic template. To deal with the point sources, we fit all $\gamma$-ray point sources with TS value larger than 25 in the radius of interest, and use the catalog spectral information for sources with smaller TS. The results are summarised in Fig, 1 and Fig, 2

In Fig 1 are shown the spectral energy distributions (SED), $E^{2} \mathrm{~d} N / \mathrm{d} E$, characterising the energy spectra of $\gamma$-rays from three different regions of the Galactic Disk. They are similar in a sense that above $2 \mathrm{GeV}$ all three can be approximated by a power-law distribution, $\mathrm{d} N / \mathrm{d} E \propto$ $E^{-\Gamma}$, and below $2 \mathrm{GeV}$ they are flat. At the same time, these spectra differ from each other, namely at high energies they are characterised by different values of the photon index $\Gamma$. The strong variation of the spectral index in the galactic plane is demonstrated in Fig,2. Note that while at energies above $2 \mathrm{GeV}$ the photon index $\Gamma$ mimics the power-law index of the spectrum of parent protons, the hardening of the spectrum below $2 \mathrm{GeV}$ is explained by the specifics of the kinematics of decay of secondary $\pi^{0}$ mesons from $p p$ interactions, and, partly, by contributions of leptonic components of $\gamma$-rays from bremsstrahlung and inverse Compton scattering of relativistic electrons [see e.g. 8]. The main conclusion from Fig 1 and Fig 2 is that the photon indices of the diffuse $\gamma$-ray emission associated with the pion-decay $\gamma$-rays, increases significantly from 2.4 in the GC direction to 2.8 in the anti-GC direction. This can be interpreted as a tendency of hardening of the spectrum of CR protons and nuclei in the inner Galaxy.

For a cross check, we also apply gtlike in the analysis with dust templates. The results appear to be consistent with that from the likelihood analysis. We also check whether this effect appears also at higher latitudes. To do this, we performed the same analysis in the latitude intervals $5^{\circ}<|b|<10^{\circ}$ and $10^{\circ}<|b|<15^{\circ}$. The results are shown in Fig 3 and Fig 4 . One can see that for large latitudes the power-law photon index is nearly the same in all directions. Thus the effect of hardening of the $\gamma$-ray spectrum is relevant only to the Galactic plane.

\section{B. $\gamma$-ray emissivity in the gas rings}

To investigated further the tendency of spectral hardening, we used, instead of dust opacity maps, the HI

\footnotetext{
1 http://fermi.gsfc.nasa.gov/ssc

2 gll_iem_v06.fit, available at/http://fermi.gsfc.nasa.gov/ssc/data/access/lat/BackgroundModels.html

3 http://galprop.stanford.edu/webrun/ $\quad{ }^{4}$ http://pypi.python.org/pypi/iminuit
} 


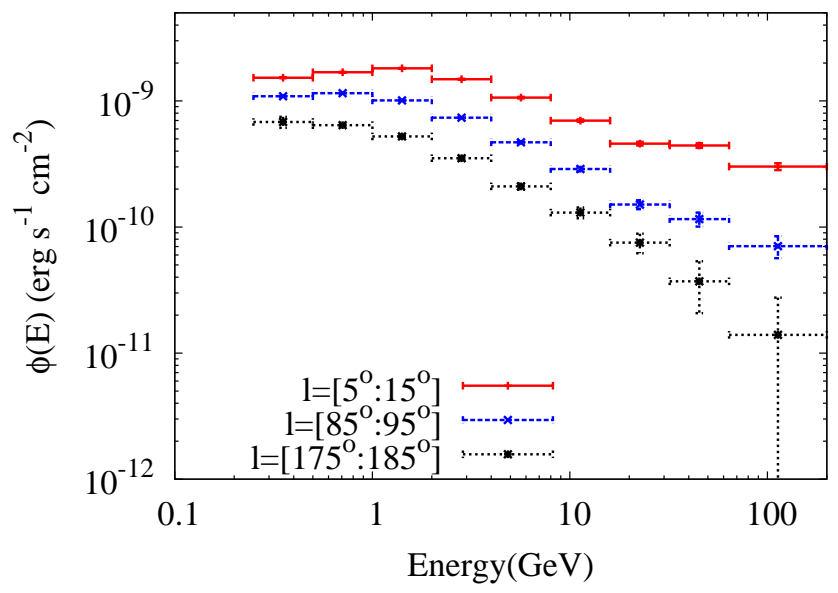

FIG. 1: The spectral energy distribution (SED) of the galactic diffuse $\gamma$-ray emission associated with the dust opacity in three different directions.

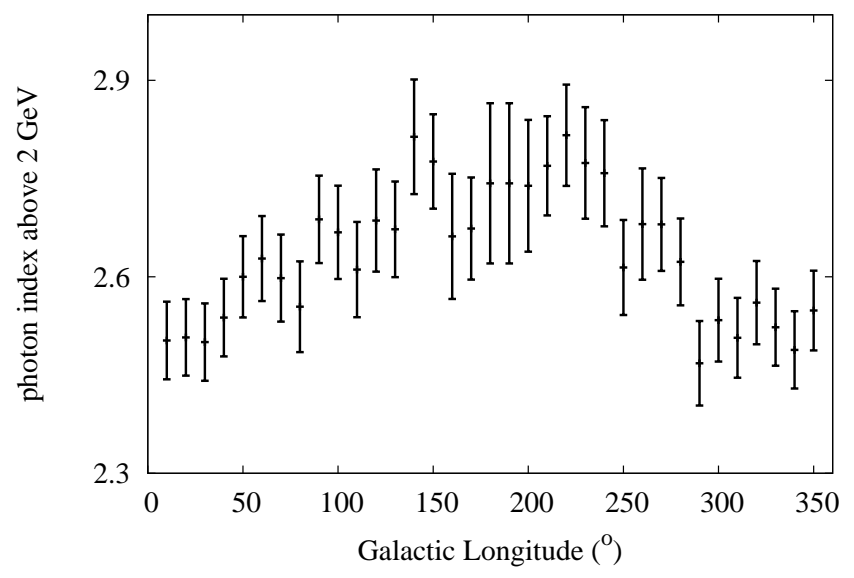

FIG. 2: The distribution of the power-law photon index of the galactic diffuse $\gamma$-ray emission associated with the dust opacity over the galactic longitudes integrated for the interval $|b|<5^{\circ}$.

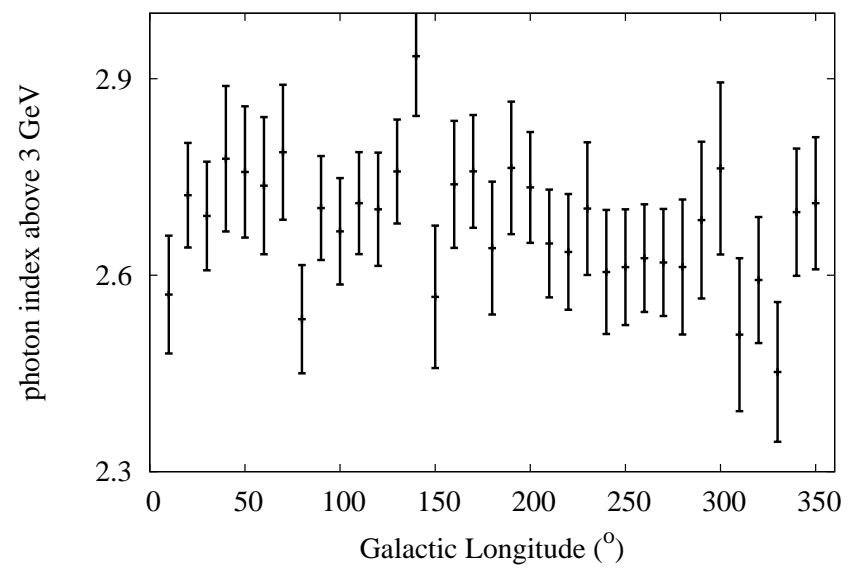

FIG. 3: The same as in Fig 2 but for the latitude interval $5^{\circ}<|b|<10^{\circ}$. 


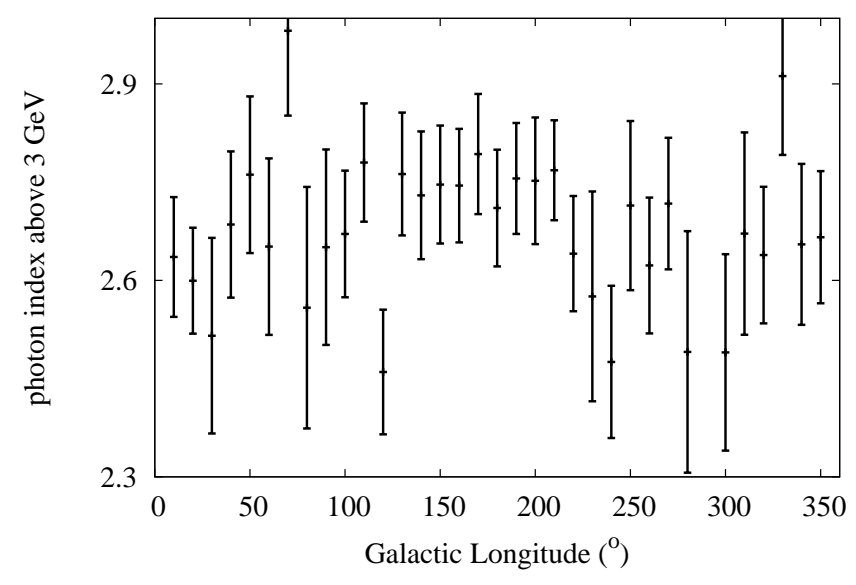

FIG. 4: The same as in Fig 2 but for the latitude interval $10^{\circ}<|b|<15^{\circ}$.

TABLE I: $X_{\mathrm{CO}}$ derived from the likelihood fit.

\begin{tabular}{l|l}
\hline Ring & $X_{\mathrm{CO}}$ \\
\hline $1 \mathrm{kpc}<\mathrm{r}<2 \mathrm{kpc}$ & $1.5 \pm 0.2 \times 10^{2 \sigma}$ \\
\hline $2 \mathrm{kpc}<\mathrm{r}<4 \mathrm{kpc}$ & $1.4 \pm 0.2 \times 10^{20}$ \\
\hline $4 \mathrm{kpc}<\mathrm{r}<6 \mathrm{kpc}$ & $0.5 \pm 0.2 \times 10^{20}$ \\
\hline $6 \mathrm{kpc}<\mathrm{r}<8 \mathrm{kpc}$ & $1.4 \pm 0.2 \times 10^{20}$ \\
\hline $8 \mathrm{kpc}<\mathrm{r}<12 \mathrm{kpc}$ & $2.0 \pm 0.2 \times 10^{20}$ \\
\hline $12 \mathrm{kpc}<\mathrm{r}<25 \mathrm{kpc}$ & $3.7 \pm 0.4 \times 10^{20}$ \\
\hline
\end{tabular}

and $\mathrm{CO}$ data which contain information on distances. The data are divided into 6 rings around the GC: $[1,2]$, $[2,4],[4,6],[6,8],[8,12]$ and $[12,25] \mathrm{kpc}$. To account for the so-called "dark gas", we also included the "dark" gas template as described in Sec.2. To keep the calculation time at a reasonable level, we fix both the absolute fluxes and photon indices of all point sources to the values from the fit in the dust template fitting, or to the catalog values. The difference in this two cases is small (we included it in the systematic errors of the final data points). To reduce further the degree of freedom in the likelihood fit, we first fit the gamma ray emissivity in the $2-4 \mathrm{GeV}$ energy interval, where we deal with adequate photon statistics combined with a reasonably good PSF. From the results of fitting in this energy band, we derive the factor $X_{\mathrm{CO}}$ by assuming that the $\gamma$-ray emissivity per $H_{2}$ is twice of the emissivity per neutral hydrogen atom . Then we fix this factor and apply the likelihood fitting for other energy intervals. The results are shown in Fig 5 , Fig 6 and Fig[7 The fitted $X_{\mathrm{CO}}$ are listed in Table!

The results are consistent with the conclusion based on the analysis of dust templates described in the previous section. The derived power-law indices imply a clear tendency of spectral softening toward the outer Galaxy. Namely, the power-law photon index above $2 \mathrm{GeV}$ varies from $\approx 2.4$ in the innermost ring to 2.8 in the outermost one. Furthermore, it can be seen in Fig.7 that the normalised $\gamma$-ray emissivity (per $\mathrm{H}$-atom) also varies with
TABLE II: Energy density of CRs in different rings.

\begin{tabular}{l|l|l}
\hline Ring & $\epsilon_{\mathrm{CR}}(1-10 \mathrm{GeV})\left(\mathrm{eV} / \mathrm{cm}^{3}\right)$ & $\epsilon_{\mathrm{CR}}(\dot{i} 10 \mathrm{GeV})$ \\
\hline$r<8 \mathrm{kpc}$ & $1.09 \pm 0.30$ & $0.67 \pm 0.13$ \\
\hline$r>8 \mathrm{kpc}$ & $0.37 \pm 0.14$ & $0.12 \pm 0.04$ \\
\hline $4 \mathrm{kpc}<\mathrm{r}<8 \mathrm{kpc}$ & $1.60 \pm 0.45$ & $1.00 \pm 0.15$ \\
\hline AMS-02 & $0.13 \pm 0.001$ & $0.09 \pm 0.001$ \\
\hline GeV excess & $0.02 \pm 0.01$ & $0.02 \pm 0.01$ \\
\hline
\end{tabular}

the distance to the GC. Since the normalised $\gamma$-ray emissivity is proportional to the CR density, Fig 7 can be treated as a radial profile of the $\mathrm{CR}$ density. One can see that the CR density achieves its maximum in the ring $[4,6] \mathrm{kpc}$. Note that the peak is coincident with the peaks of distributions of SNRs [11] and OB stars [12]. Interestingly, the peak coincides with the minimum of the conversion factor $X_{\mathrm{CO}}$ which appears in the same ring (see Table $\llbracket$ ). Note that the small values of the $X_{\mathrm{CO}}$ factor in the inner Galaxy has been independently derived in Ackermann et al. [13]. The observed gradient in metallicity across the Galaxy [14] also supports the low values of $X_{\mathrm{CO}}$. Yet, such a coincidence between the CR density and the conversion factor $X_{\mathrm{CO}}$ seems, to some extent, suspicious. Thus, one cannot exclude that the peak in the $\gamma$-ray emissivity could be caused by the underestimation of the gas density in this region. For comparison, in fig 8 we show the $\gamma$-ray emissivity derived under an $a d$ hoc assumption of constant conversion factor $X_{\mathrm{CO}}$ in all rings fixed to the value $2.0 \pm 0.2 \times 10^{20}$. One can see that in this case the maximum in the $\gamma$-ray emissivity in the 4-6 kpc ring disappears.

\section{DISCUSSION}

In this paper we present the results of our analysis of the diffuse galactic $\gamma$-ray emission based on the FermiLAT $\gamma$-ray data combined with the HI, CO and the dust- 


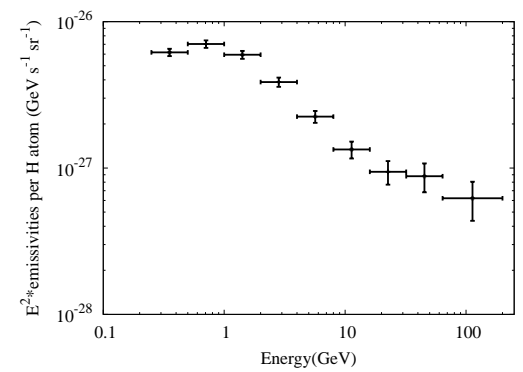

(a) $1-2 \mathrm{kpc}$

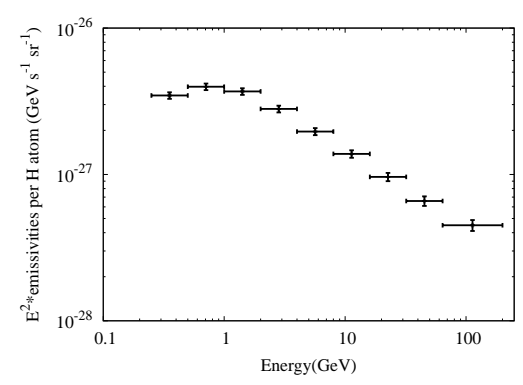

(d) $6-8 \mathrm{kpc}$

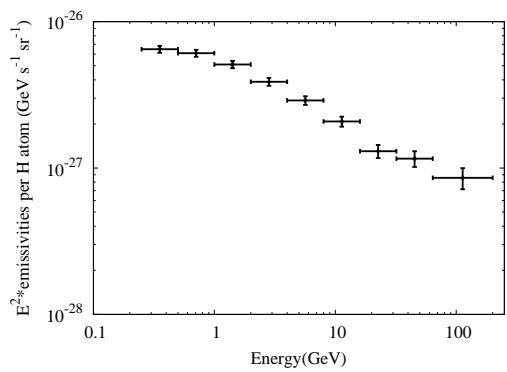

(b) $2-4 \mathrm{kpc}$

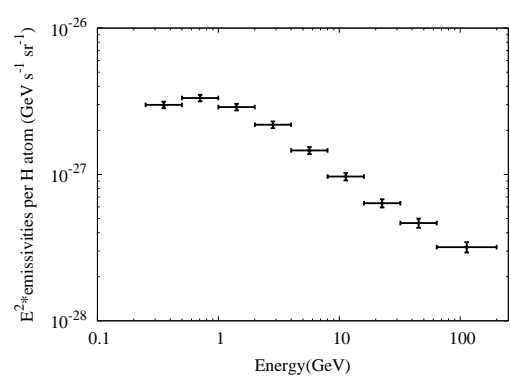

(e) $8-12 \mathrm{kpc}$

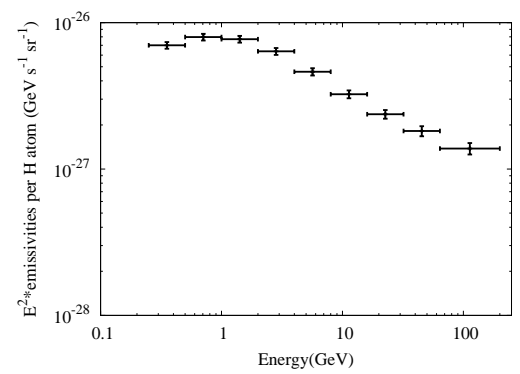

(c) $4-6 \mathrm{kpc}$

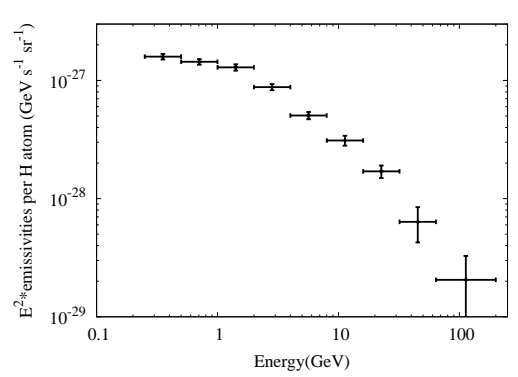

(f) $12-25 \mathrm{kpc}$

FIG. 5: The SED of galactic diffuse $\gamma$-ray emission associated with the gas in different rings around the GC.

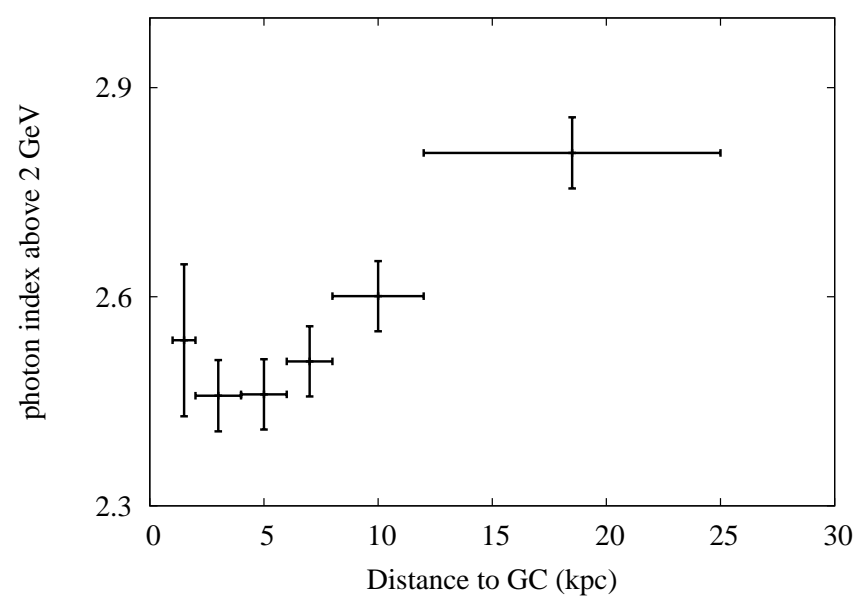

FIG. 6: The distribution of the photon index of the galactic diffuse gamma ray emission associated with the gas in different rings.

opacity maps. The energy range of detected $\gamma$-rays from $100 \mathrm{MeV}$ to $200 \mathrm{GeV}$ allows derivation of detailed spectral and spatial distributions of CRs over almost four energy decades, from mildly relativistic (sub-GeV) to ultrarelativistic (multi-TeV) energies.

The results described in the previous sections demonstrate strong variations of both the energy spectra and the absolute fluxes of $\gamma$-rays throughout the entire galactic plane $\left(0^{\circ}<l<360^{\circ}\right.$ and $\left.|b|<5^{\circ}\right)$. The energy spectra of $\gamma$-rays arriving from the directions of inner Galaxy appear significantly harder compared to the spectra of radiation from outer parts of the Galaxy. The tendency of the spectral change is clearly seen in Fig 1 and Fig, 2 , This conclusion agrees with the recent analysis of the Fermi LAT data reported in ref. Casandjian [5], and, with some reservation, with the old results reported by the EGRET team [18] 5. A hard diffuse $\gamma$-ray spectrum

\footnotetext{
${ }^{5}$ Note that although the EGRET result later has been criticised, and the very existence of the "GeV bump" has been discarded by the community, one should point out that in general terms the latter is in reasonable agreement with the recent Fermi-LAT data.
} 


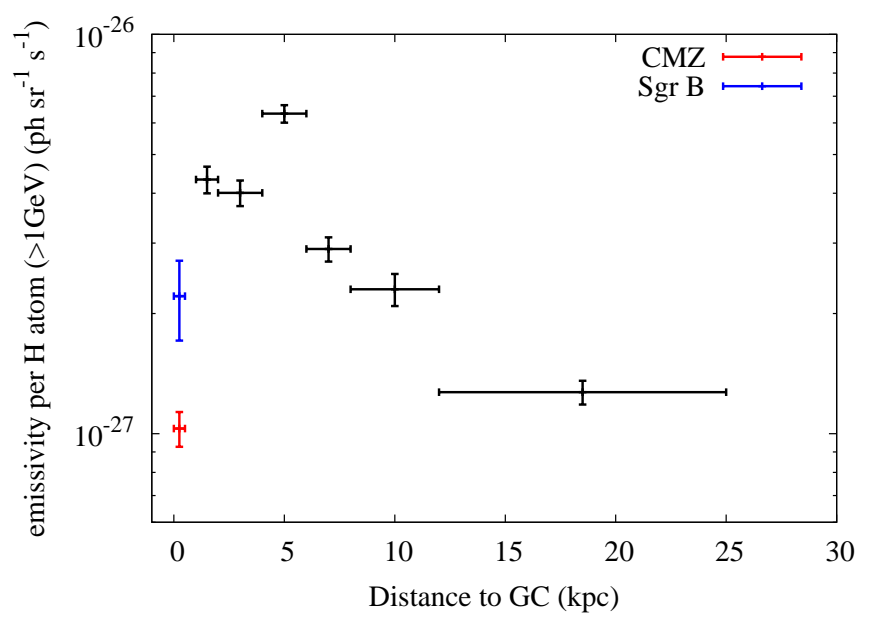

FIG. 7: Normalised per H-atom gamma ray emissivities above $1 \mathrm{GeV}$ in different rings by using the best fit conversion factor $X_{\mathrm{CO}}$ as shown in Tab.1. The points close to the GC are taken from analysis of the $\gamma$-ray luminosity of the entire Central Molecular Zone [5] and from the analysis of the emissivity of the giant molecular complex Sgr B2 [15].

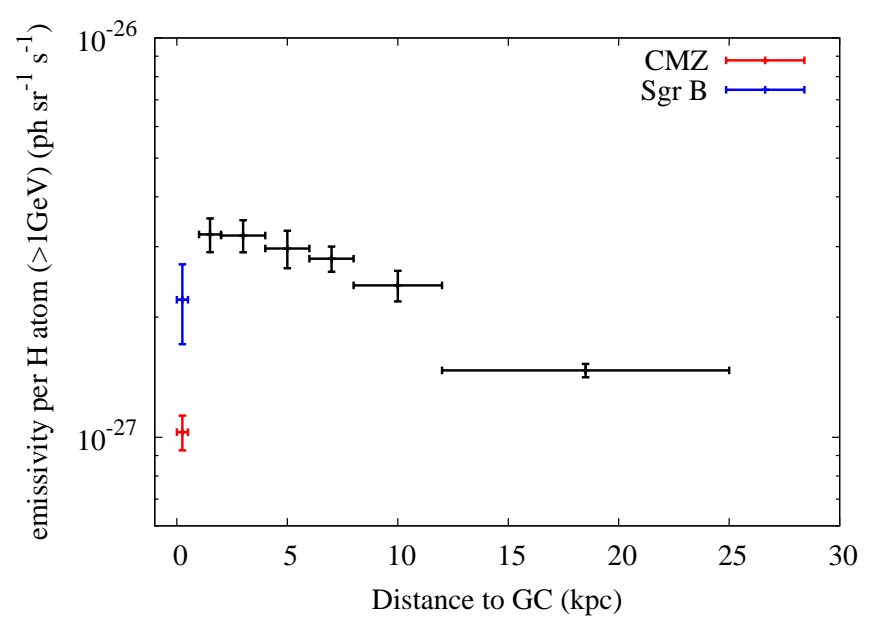

FIG. 8: The same as in Fig[7 but for the conversion factor $X_{\mathrm{CO}}$ fixed at the local value $2.0 \pm 0.2 \times 10^{20}$.

has been recently reported also in Neronov and Malyshev [19] who claimed a universal photon index close to 2.45 throughout the entire Galactic Disk. However, this conclusion based on a rather limited range of galactic longitudes $\left(|l| \leq 90^{\circ}\right)$, is misleading. The results shown in Fig 1 and Fig 2 reveal a non-negligible spectral variation throughout the galactic plane. In particular, while the $\gamma$-ray spectrum in the inner parts of the Galaxy is significantly harder than the spectrum of locally measured $\mathrm{CRs}$, the latter is quite close to the spectrum of $\gamma$-rays (and, consequently, to the spectrum of parent protons) from the anti-centre direction (where the Solar system is located). Moreover, the comparison of Fig 2 with Fig 3 and Fig, makes it clear that the spectral hardening takes place only in the galactic plane.

In general, the spectral hardening of $\gamma$-rays could be caused by contamination of the truly diffuse flux of $\gamma$ - ray (i.e. the ones produced in interactions of CRs) by discrete $\gamma$-ray sources concentrated in the inner Galaxy. We believe that the discrete sources have been carefully treated in our analysis. Nevertheless, we cannot exclude the possibility that a new population of weak but numerous hard-spectrum $\gamma$-ray sources, which have not been resolved by Fermi-LAT, significantly contribute to the truly diffuse $\gamma$-ray background.

Alternatively, the hardening could be caused, in principle, by undervaluation of the contribution of the IC component of $\gamma$-rays in the inner Galaxy. In this work, a spatial IC template based on the calculations with the GALPROP code [9], was used to model the IC emission. Although the interstellar radiation fields (ISRF) are poorly constrained, their enhancement in the inner Galaxy (compared to the values provided by GALPROP) hardly can exceed a factor of two or three. Meanwhile, 


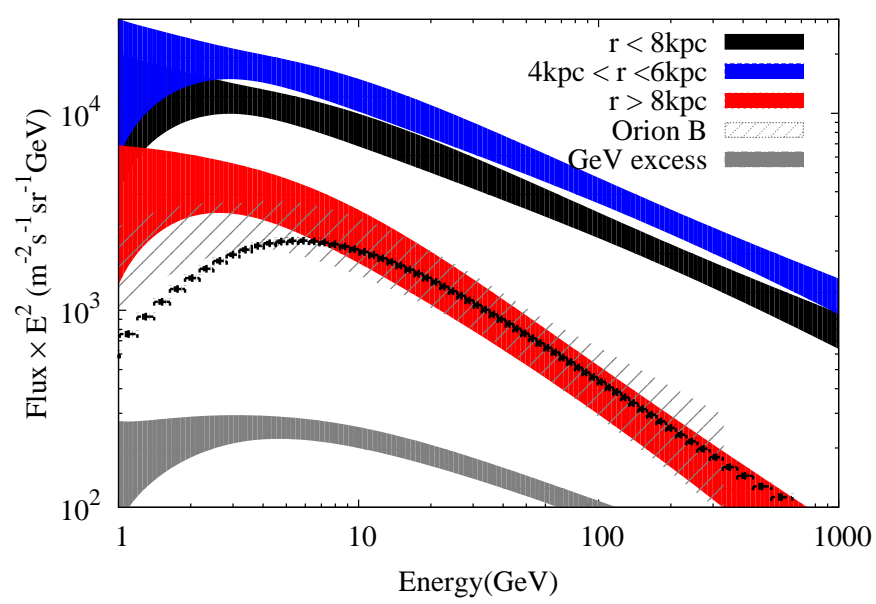

FIG. 9: The CR proton spectra in the inner $(r \leq 8 \mathrm{kpc})$ and outer $(r \geq 8 \mathrm{kpc})$ regions, as well as in the $4-6 \mathrm{kpc}$ ring derived from $\gamma$-ray emissivities presented in Fig 7 . Also are shown the proton spectra derived from the $\gamma$-ray measurements of the nearby molecular cloud Orion B [16]. and from the low-energy $\gamma$-ray component called "GeV excess". The direct measurements of the CR proton spectrum are from the AMS-02 collaboration report [17], which are shown as black squares.

at multi-GeV energies the component of $\pi^{0}$-decay $\gamma$-rays strongly, by on order of magnitude, dominates over the IC contribution calculated for the "standard" fluxes of ISRF [see 8]. Consequently, the interpretation of the spectral hardening of $\gamma$-rays by an enhanced IC contribution, seems rather unlikely.

Thus, we may conclude, although not without the caveat concerning the possible non-negligible contribution of unresolved $\gamma$-ray sources, that the major fraction of the diffuse $\gamma$-ray flux at $\mathrm{GeV}$ energies arises from interactions of CR protons and nuclei with the interstellar gas (through the production and decay of the secondary neutral pions). In the framework of this interpretation, the hardening of the $\gamma$-ray spectrum in the inner Galaxy can be explained by the concentration of CR accelerators in the inner Galaxy and/or by the CR propagation effects [see e.g. 20]. The radial distributions of the normalised $\gamma$-ray emissivity (Fig 7 and Fig 8), as well as the radial dependence of the photon index (Fig,6), contain unique information regarding the spatial distribution of CR accelerators.

If the distinct maximum in the radial distribution of the $\gamma$-ray emissivity in Fig 7 calculated for the values of the conversion factor $X_{\mathrm{CO}}$ from Table 1 , is real, it is straightforward to assume that the CR accelerators are concentrated in the 4-6 kpc ring. The continuous injection of CRs into the interstellar medium can explain the gradual drop of the $\gamma$-ray emissivity in both directions - towards the GC and to the periphery of the Galaxy (see Fig.7). While in the homogeneous and spherically symmetric medium we expect $1 / r$ type distribution for the CR density [see e.g. 8], radial dependence of the diffusion coefficient and its possible anisotropic character could result in a deviation from the $1 / r$ dependence. Detailed numerical treatment of the $\mathrm{CR}$ propagation, and the comparison of theoretical predictions with the results presented in Fig:7 and Fig 6 , would provide an important information on the spatial distribution of sources of CRs, on the character of their propagation in the interstellar magnetic fields, on the total CR injection rate, etc.

What concerns the harder energy spectrum of CRs which are currently confined in the $4-6 \mathrm{kpc}$ ring, compared to their spectrum in outskirts of the Galaxy, a possible reason of this effect could be an intrinsic feature of particle accelerators in the ring. For example, one may speculate that the sources in the inner Galaxy accelerate CRs with harder spectra than the CR accelerators do in the outer Galaxy. An alternative explanation of of this effect could be related to the specifics of propagation of particles in the inner Galaxy. In particular, it could be caused by different rates of escape of CRs in different parts of the Galaxy. For example, the stronger galactic wind in the inner Galaxy, caused by a higher local pressure, may result in the transport of CRs which at low energies might be dominated by advection rather than diffusion. Consequently, at low energies the shape of the CR spectrum in the inner Galaxy would not suffer deformation [8], while in the outer Galaxy the energydependent diffusion could lead to significant steepening of the original (acceleration) spectrum. Apparently, these two scenarios need further observational and theoretical inspections. In particular, it would be important to perform independent measurements of the CR spectra from individual massive clouds which can serve as CR barometers [21 23]. Such measurements, already have been conducted for the nearby molecular clouds in the Gould Belt [16] and local Hi regions [24, 25]. They have revealed absolute fluxes and energy spectra of CRs which are in a good agreement with the results of this paper for the outer Galaxy, and with the recent direct measurements 
of CR protons reported by the AMS-02 collaboration [17] (see Fig 9).

Finally, we note that the 4-6 kpc ring seems to be a rather natural place for concentration of CR accelerators, given that the potential CR source populations, such as pulsars, OB stars and SNRs, have radial profiles which also shows a maximum at several kpc from the Galatic Centre [11, 12]. Yet, it sounds somewhat suspicious that the reason of the maximum in the $\gamma$-ray luminosity in the $4-6 \mathrm{kpc}$ ring is the radial dependence of the conversion factor $X_{\mathrm{CO}}$ which has a sharp minimum exactly in the same $4-6 \mathrm{kpc}$ ring (see Table.I). Whether we deal with an accidental coincidence or there are some deeper reasons for such a coincidence, this is a question which is beyond the framework of the present paper. On the other hand, if we assume a smoother radial-dependence of the conversion factor $X_{\mathrm{CO}}$, the corresponding radial distribution of the $\gamma$-ray emissivity would become quite different from the distribution in Fig 7 . For example, the (ad hoc assumed) constant conversion factor $X_{\mathrm{CO}}$ would remove the maximum of the $\gamma$-ray emissivity at $4-6 \mathrm{kpc}$ (see Fig, 8). Instead, it would result in a continuous increase of the CR density towards the GC. In this case, the GC itself would be the most natural place for CR production, given that some other $\gamma$-ray phenomena like the $\mathrm{TeV} \gamma$-ray emission of the Central Molecular Zone [26, 27], the so-called "GeV excess" [28 32], the Fermi Bubbles [33], are most likely linked, in one way or another, to the GC. To sustain the observed density of CRs, the average $\mathrm{CR}$ production over the last $10^{7}$ years should proceed at the rate of $10^{41} \mathrm{erg} / \mathrm{s}$. The only source in this compact region which could provide (in principle) such a power in relativistic particles is the Supermassive Black
Hole in the dynamical centre of our Galaxy. In this scenario, the significant reduction of the $\mathrm{CR}$ density in the very central (within $100 \mathrm{pc}$ ) region of the GC can be explained by the rather low activity of the source over the last $10^{3-4}$ years (the characteristic timescales of propagation of CRs on100 pc scales), and/or the fast removal of CRs from this region, for example, due to convection.

Independent of the origin of the parent protons, the results of this paper demonstrate that the concept of the so-called "sea" of galactic CRs, which assumes nearly uniform spatial and energy distributions of CRs in the Galactic Disk, is a very rough approximation which perhaps can be used for zeroth-order estimates of global parameters, but not for a quantitative description of the CR production and transport throughout the Galaxy. This is demonstrated in Fig 9 where we show the average energy spectra of CR protons at different radial distances from the GC. They are derived from $\gamma$-ray emissivities presented in Fig 7 (in calculations we take into account the enhancement of the $\gamma$-ray production rate due to the CR nuclei by a factor of 1.8 [34]). In the same figure we show the CR proton fluxes in our neighbourhood from direct measurements of AMS-02 [17], and from the $\gamma$ ray observations in the nearby molecular cloud Orion B2 [16]. The flux of the $\mathrm{CR}$ proton component responsible for the so-called "GeV-excess" in the central several kpc region of the inner Galaxy is also shown. Note that at low energies we may expect significant contribution from the bremsstrahlung and IC components of $\gamma$-rays, therefore the curve should be considered as an upper limit. In any case, the contribution of particles responsible for the "GeV-excess" is well below the contribution of the main CR component.
[1] L. O. Drury, Astropart.Phys. 39-40, 52 (2012) arXiv:1203.3681 [astro-ph.HE]

\begin{tabular}{|ll|l|}
\hline$[2]$ P. & Blasi, & A\&A Rev. 21, 70 (2013)
\end{tabular} arXiv:1311.7346 [astro-ph.HE].

[3] P. Cristofari, S. Gabici, S. Casanova, R. Terrier, and E. Parizot, MNRAS 434, 2748 (2013), arXiv:1302.2150 [astro-ph.HE].

[4] T. M. Dame, D. Hartmann, and P. Thaddeus, ApJ 547, 792 (2001), arXiv:astro-ph/0009217.

[5] J.-M. Casandjian, ArXiv e-prints arXiv:1502.07210 [astro-ph.HE]

[6] Planck Collaboration, P. A. R. Ade, N. Aghanim, M. Arnaud, M. Ashdown, J. Aumont, C. Baccigalupi, A. Balbi, A. J. Banday, R. B. Barreiro, and et al., A\&A 536, A19 (2011), arXiv:1101.2029 [astro-ph.GA].

[7] D. P. Clemens, ApJ 295, 422 (1985).

[8] F. A. Aharonian and A. M. Atoyan, A\&A 362, 937 (2000), astro-ph/0009009

[9] A. E. Vladimirov, S. W. Digel, G. Jóhannesson, P. F. Michelson, I. V. Moskalenko, P. L. Nolan, E. Orlando, T. A. Porter, and A. W. Strong, Computer Physics Communications 182, 1156 (2011), arXiv:1008.3642 [astro-ph.HE]
[10] The Fermi-LAT Collaboration, ArXiv e-prints (2015), arXiv:1501.02003 [astro-ph.HE]

[11] G. L. Case and D. Bhattacharya, ApJ 504, 761 (1998), astro-ph/9807162

[12] L. Bronfman, S. Casassus, J. May, and L.-A. Nyman, A\&A 358, 521 (2000), astro-ph/0006104

[13] M. Ackermann, M. Ajello, W. B. Atwood, L. Baldini, J. Ballet, G. Barbiellini, D. Bastieri, K. Bechtol, R. Bellazzini, B. Berenji, R. D. Blandford, et al., ApJ 750, 3 (2012), arXiv:1202.4039 [astro-ph.HE].

[14] J. Y. Cheng, C. M. Rockosi, H. L. Morrison, R. A. Schönrich, Y. S. Lee, T. C. Beers, D. Bizyaev, K. Pan, and D. P. Schneider, ApJ 746, 149 (2012), arXiv:1110.5933

[15] R.-z. Yang, D. I. Jones, and F. Aharonian, A\&A 580, A90 (2015), arXiv:1410.7639 [astro-ph.HE],

[16] R.-z. Yang, E. de Oña Wilhelmi, and F. Aharonian, A\&A 566, A142 (2014), arXiv:1303.7323 [astro-ph.HE]

[17] M. Aguilar, D. Aisa, B. Alpat, A. Alvino, G. Ambrosi, K. Andeen, L. Arruda, N. Attig, P. Azzarello, A. Bachlechner, and et al., Physical Review Letters 115, 211101 (2015).

[18] S. D. Hunter, D. L. Bertsch, J. R. Catelli, T. M. Dame, 
S. W. Digel, B. L. Dingus, J. A. Esposito, C. E. Fichtel, R. C. Hartman, G. Kanbach, D. A. Kniffen, Y. C. Lin, H. A. Mayer-Hasselwander, P. F. Michelson, C. von Montigny, R. Mukherjee, P. L. Nolan, E. Schneid, P. Sreekumar, P. Thaddeus, and D. J. Thompson, ApJ 481, 205 (1997).

[19] A. Neronov and D. Malyshev, ArXiv e-prints (2015), arXiv:1505.07601 [astro-ph.HE].

[20] D. Gaggero, A. Urbano, M. Valli, and P. Ullio, Phys. Rev. D 91, 083012 (2015) arXiv:1411.7623 [astro-ph.HE],

[21] F. A. Aharonian, Ap\&SS 180, 305 (1991)

[22] F. A. Aharonian, Space Sci. Rev. 99, 187 (2001), astro-ph/0012290

[23] S. Casanova, F. A. Aharonian, Y. Fukui, S. Gabici, D. I. Jones, A. Kawamura, T. Onishi, G. Rowell, H. Sano, K. Torii, and H. Yamamoto, PASJ 62, 769 (2010), arXiv:0904.2887 [astro-ph.HE].

[24] A. A. Abdo, M. Ackermann, M. Ajello, W. B. Atwood, M. Axelsson, L. Baldini, J. Ballet, G. Barbiellini, et al., ApJ 703, 1249 (2009), arXiv:0908.1171 [astro-ph.HE].

[25] J.-M. Casandjian, ApJ 806, 240 (2015) arXiv:1506.00047 [astro-ph.HE].

[26] F. Aharonian, A. G. Akhperjanian, A. R. BazerBachi, M. Beilicke, W. Benbow, D. Berge, K. Bernlöhr,
C. Boisson, O. Bolz, V. Borrel, Braun, et al., Nature 439, 695 (2006), astro-ph/0603021

[27] H. E. S. S. Collaboration, A. Abramowski, F. Aharonian, F. Ait Benkhali, A. G. Akhperjanian, E. O. Angüner, M. Backes, A. Balzer, Y. Becherini, J. Becker Tjus, and et al., Nature accepted.

[28] L. Goodenough and D. Hooper, ArXiv e-prints (2009), arXiv:0910.2998 [hep-ph]

[29] K. N. Abazajian and M. Kaplinghat, $\quad$ Phys. Rev. D 86, 083511 (2012) arXiv:1207.6047 [astro-ph.HE]

[30] T. Daylan, D. P. Finkbeiner, D. Hooper, T. Linden, S. K. N. Portillo, N. L. Rodd, and T. R. Slatyer, ArXiv e-prints (2014), arXiv:1402.6703 [astro-ph.HE]

[31] O. Macias and C. Gordon, Phys. Rev. D 89, 063515 (2014). arXiv:1312.6671 [astro-ph.HE]

[32] F. Calore, I. Cholis, and C. Weniger, J. Cosmology Astropart. Phys. 3, 038 (2015) arXiv:1409.0042

[33] M. Su, T. R. Slatyer, and D. P. Finkbeiner, ApJ 724, 1044 (2010), arXiv:1005.5480 [astro-ph.HE],

[34] E. Kafexhiu, F. Aharonian, A. M. Taylor, and G. S. Vila, Phys. Rev. D 90, 123014 (2014), arXiv:1406.7369 [astro-ph.HE] 